\title{
El uso de dispositivo intrauterino con cobre no está asociado al riesgo de infertilidad tubaria en mujeres nuligestas
}

Use of copper intrauterine devices and the risk of tubal infertility among nulligravid women. Hubacher $\mathrm{D}$, Lara-Ricalde $\mathrm{R}$, Taylor $D$. $N$ Engl $J$ Med 2001; 345:561-567.

\section{Objetivo}

Determinar si el uso de DIU (dispositivo intrauterino) con cobre en mujeres nuligestas es un factor de riesgo para la oclusión tubaria e infertilidad.

\section{Diseño}

Estudio de casos y controles. *

Lugar

Tres hospitales públicos en México.

\section{Participantes}

Se incluyeron 1311 mujeres infértiles (358 mujeres con oclusión tubaria, que fueron los casos; y 953 controles). Se reclutó un segundo grupo control con 584 mujeres embarazadas.

\section{Evaluación de factores de riesgo}

Las participantes respondieron acerca del uso previo de métodos anticonceptivos, relaciones sexuales previas e historia de infección del tracto genital inferior.

\section{Medición de Resultados Principales}

Atodas las mujeres nuligestas se les practicó una histerosalpingografía para diagnóstico de oclusión tubaria. Se consideraron como posibles predictores de infertilidad tubaria el uso de DIU con cobre como variable de exposición primaria y otras variables como la presencia de anticuerpos para Chlamydia Trachomatis, el número de parejas sexuales, la presencia de síntomas ginecológicos que sugieran infección, el uso de otros métodos anticonceptivos, el nivel económico, el nivel de educación, el empleo y la existencia de relaciones sexuales durante la adolescencia.

\section{Resultados}

El uso previo de DIU con cobre, no se asoció con un incremento en el riesgo de oclusión tubaria, tanto en el análisis que incluyó a las mujeres infértiles en el grupo control (OR 1.0, IC 95\% 0.6-1.6) como cuando se asignó a las mujeres embarazadas como grupo control (OR 0.7, IC 95\% 0.4-1.2).

La infertilidad tubaria no se asoció con la duración del uso de DIU, con las razones por las que se extrajo el mismo ni con la presencia o ausencia de problemas ginecológicos relacionados con su uso. La presencia de anticuerpos contra Chlamydia Trachomatis sí se asoció con infertilidad.

\section{Conclusión}

El uso previo de DIU con cobre no se asoció a un aumento en el riesgo de oclusión tubaria en mujeres nuligestas, mientras que si lo hizo la presencia de anticuerpos contra Chlamydia Trachomatis.

\section{Comentario}

Más de cien millones de mujeres en todo el mundo usan DIU como método anticonceptivo. Apesar de su eficacia, la preocupación sobre si su utilización puede causar infección pelviana e infertilidad ha marginado notablemente su uso en algunos países.

Los investigadores de este estudio seleccionaron mujeres nuligestas infértiles con oclusión tubaria y a dos grupos controles (mujeres infértiles sin oclusión tubaria y mujeres embarazadas), comparándolos en cuanto al uso previo de DIU con cobre y otros factores de riesgo potenciales de infertilidad. También evaluaron en las mujeres la presencia de anticuerpos contra Chlamydia Trachomatis, el factor más importante relacionado con infertilidad.

Los autores no encontraron aumento en el riesgo de oclusión tubaria en las mujeres que habían usado previamente DIU. En contraste, existió una fuerte asociación entre la infección previa por Chlamydia Trachomatis y la infertilidad.

Este estudio se contrapone a reportes previos que sugerían una relación directa entre las mujeres que habían usado DIU con cobre y la infertilidad debido a oclusión tubaria. Estos dos estudios de casos y controles publicados hace 16 años, reportaron RR para infertilidad de $1.3^{1}$ y 1.52 respectivamente, pero en ambos los intervalos de confianza eran amplios, por lo que ninguno demostró en forma significativa la asociación entre el uso de DIU y el riesgo de infertilidad. Posiblemente este incremento en el riesgo relativo de infertilidad tubaria en mujeres portadoras de DIU, fue debido a la falta de medición del efecto confundidor* de la exposición a Chlamydia Trachomatis, ya que al momento de realizarse éstos trabajos se desconocía su importante rol como causante de infertilidad tubaria.

Sin importar el tipo de anticoncepción elegida, la creciente prevalencia de Chlamydia y otros patógenos de transmisión sexual, sugiere que el uso de condones debería ser el método anticonceptivo de elección. De todas formas las mujeres nuligestas, quienes no se encuentren en riesgo de padecer enfermedades de transmisión sexual, son candidatas para el uso de DIU con cobre. Éste podría ser entre los métodos anticonceptivos reversibles, el más seguro, efectivo y barato. Por lo tanto: ¿es tiempo de pedirle perdón al DIU?. ${ }^{3}$

Dr. Julio Matz [ Servicio Medicina Familiar. CEMIC ]

Referencias
1- Primary tubal infertility in relation to the use of an intrauterine device. Dalling J, Weiss N, Metch B. N Engl J Med 1985; $312: 937-941$.
2- Tubal infertility and the intrauterine device. Cramer D, Schiff I, Schoembaum S. N Engl J Med 1985; 312: 941-947.

3- Time to pardon the IUD?. Darney P. N Engl J Med 2001; 345: 608-610. 